\title{
TRANSCENDENTAL MEROMORPHIC SOLUTIONS OF A TYPE OF $q$-DIFFERENCE COMPOSITE FUNCTIONAL EQUATIONS
}

\section{SHUXIN HAN ${ }^{1}$, YUE WANG ${ }^{1}$, JIANYING LIANG ${ }^{1}$ and HUA WANG ${ }^{2}$}

${ }^{1}$ College of Mathematics and Statistics

Hebei University of Economics and Business

Shijiazhuang, 050061

P. R. China

e-mail:wy2006518@163.com

${ }^{2}$ Shijiazhuang Tiedao University

SiFang College

Shijiazhuang, 050061

P. R. China

\begin{abstract}
In this paper, we will investigate transcendental meromorphic solutions of a type of $q$-difference composite functional equations, characteristic function of solutions is estimated. We obtain one interesting result. Example shows that our result is precise.
\end{abstract}

2010 Mathematics Subject Classification: 30D35, 39A12.

Keywords and phrases: $q$-difference composite functionl equation, transcendental meromorphic solution, meromorphic functions.

Received March 1, 2018; Revised March 25, 2018

(ㄷ) 2018 Scientific Advances Publishers 


\section{Introduction and Main Results}

Nevanlinna value distribution theory of meromorphic functions has been extensively applied to resolve growth (see, e.g., [1]), value distribution [1], and solvability of meromorphic solutions of linear and nonlinear differential equations (see, e.g., [2]). Considering meromorphic functions $f(z)$ in the complex plane, we assume that the reader is familiar with the standard notations and results such as $m(r, f(z))$, $n(r, f(z)), N(r, f(z))$, and $T(r, f(z))$ denote the proximity function, the non-integrated counting function, the counting function and the characteristic function of $f(z)$, respectively. For the integrated counting

function for distinct poles of $f(z)$ we use the notations $\bar{N}(r, f(z))$, the order of growth of meromorphic function $f(z)$ are denote by $\rho(f(z))$.

Given a meromorphic function $f(z)$, recall that a meromorphic function $h(z)$ is said to be a small function of $f(z)$, if $T(r, h(z))=S(r, f)$, where $S(r, f)$ is used to denote any quantity that satisfies $S(r, f)$ $=o(T(r, f))$ as $r \rightarrow \infty$, possibly outside of a set of $r$ of finite logarithmic measure.

Recently, some papers focused on complex difference equations and difference analogues of Nevanlinna's theory. Many authors ([3-11]) mainly deal with the existence or the growth of meromorphic solutions of difference equations, many results on the complex difference equations are obtained.

In 2003, Silvennoinen investigated the properties of meromorphic solutions of the following composite equations:

$$
f(p(z))=\sum_{i=0}^{m} a_{i}(z)(f(z))^{i},
$$

He obtained the following result (see [12]). 
Theorem A ([12]). Suppose that $f(z)$ be a transcendental meromorphic solution of $(*)$, where $p(z)$ is a polynomial of degree of $k \geq 2$, the coefficients $\left\{a_{i}(z)\right\}$ are meromorphic, $a_{m} \neq \equiv 0$ and $m>1$. Suppose further that

$$
T\left(r, a_{j}\right)<K T\left(r^{s}, f\right), \quad j=0,1,2, \cdots, m,
$$

where $K$ and $s$ are positive constants, and $r$ is large enough. If $s<k$, then for given $\varepsilon>0$,

$$
T(r, f)=O\left((\log r)^{\alpha+\varepsilon}\right),
$$

where

$$
\alpha=\frac{\log \left((m+1) K+\frac{m}{s}\right)}{\log \frac{k}{s}}, \text { if } 1 \leq s<k
$$

and

$$
\alpha=\frac{\log ((m+1) K s+m)}{\log k} \text {, if } s<1<k \text {. }
$$

In this paper, inspired by the ideas of the paper [12], instead of considering composite equations, we shall consider characteristic estimate and non-integrated counting function of meromorphic solutions of the following $q$-difference composite functional equations:

$$
\sum_{(i) \in I} a_{(i)}(z)(w(z))^{i_{0}}\left(w\left(q_{1} z\right)\right)^{i_{1}} \cdots\left(w\left(q_{n} z\right)\right)^{i_{n}}=\sum_{j=0}^{m} a_{j}(z)(w(p(z)))^{j}
$$

where $\left\{a_{j}(z)\right\}$ are meromorphic functions, $a_{(i)} \not \equiv 0, a_{m}(z) \neq 0, q_{i} \in C \backslash\{0\}$, $i=0,1,2, \cdots, n, p(z)$ is a polynomial of degree $k \geq 2, I$ is a finite index set. 
We obtain

Theorem 1.1. Let $w(z)$ be a zero-order transcendental meromorphic solution of (1.1), $\left\{a_{(i)}(z)\right\}$ be polynomials,

$$
T\left(r, a_{j}\right)<K T\left(r^{s}, w\right), \quad j=0,1,2, \cdots, m,
$$

where $K$ and $s$ are positive constants, $r$ is large enough. If $s<k$, then for given $\varepsilon>0$,

$$
T(r, w)=O\left((\log r)^{\alpha+\varepsilon}\right),
$$

where

$$
\alpha=\frac{\log \left((m+1) K+\frac{\lambda}{m s}\right)}{\log \frac{k}{s}}, \text { if } 1 \leq s<k,
$$

and

$$
\alpha=\frac{\log \frac{\lambda+m(m+1) K s}{m}}{\log k} \text {, if } s<1<k,
$$

where $\lambda=\max _{i \in(i)}\left\{\sum_{l=0}^{n} i_{l}\right\}$.

Remark 1.1. Compared with Theorem A, although the results are basically similar, the equations we discuss are more complex and involve $q$-difference.

Remark 1.2. The example 1.1 shows that zero order solutions and the condition $s<k$ in Theorem 1.1 cannot be removed.

Example 1.1. Let $p(z)=c_{k} z^{k}+\cdots+c_{0}, \operatorname{deg} p(z) \geq 2$,

$$
a_{i}(z)=C_{m}^{i} \frac{e^{5 z}}{\left(1+e^{p(z)}\right)^{m}}, \quad i=0,1,2, \cdots, m .
$$


Then

$$
\sum_{i=0}^{m} a_{i}(z) w(p(z))^{i}=\frac{e^{5 z}}{\left(1+e^{p(z)}\right)^{m}} \sum_{i=0}^{m} C_{m}^{i} w(p(z))^{i},
$$

$w=e^{z}$ is a transcendental meromorphic solution of the composite functional-difference equation of the form

$$
\frac{w(z)(w(2 z))^{2}}{2}+\frac{w(2 z) w(3 z)}{2}=\sum_{i=0}^{m} a_{i}(z)(w(p(z)))^{i} .
$$

In this case, $w(z)$ satisfy

$$
T(r, w(z))=\frac{r}{\pi}+O(1)
$$

However, by $k \geq 2$, we have

$$
T\left(r, a_{i}(z)\right)=(1+o(1)) \frac{m\left|c_{k}\right| r^{k}}{\pi},
$$

$\rho(w)=1$, it shows that Theorem 1.1 does not hold if $s=k$ and $\rho(w) \neq 0$.

\section{Some Lemmas}

We need the following lemmas:

Lemma 2.1 ([13]). Let $w(z)$ be a non-constant zero-order meromorphic function, and $q \in C \backslash\{0\}$. Then

$$
m\left(r, \frac{w(q z)}{w}\right)=o(T(r, w))
$$

on a set of logarithmic density 1. 
Lemma 2.2 ([14]). Let $w(z)$ be a transcendental meromorphic function of zero-order and $q \in C \backslash\{0\}$. Then

$$
\begin{gathered}
N(r, w(q z))=(1+o(1)) N(r, w(z)), \\
T(r, w(q z))=(1+o(1)) T(r, w(z)),
\end{gathered}
$$

for all $r$ on a set of logarithmic density 1.

Lemma 2.3. Let $w$ be a meromorphic function of zero order, $T\left(r, a_{(i)}\right)=o(T(r, w))$

$$
\Omega(z, w)=\sum_{(i) \in I} a_{(i)}(z)(w(z))^{i_{0}}\left(w\left(q_{1} z\right)\right)^{i_{1}} \cdots\left(w\left(q_{n} z\right)\right)^{i_{n}} .
$$

Then

$$
T(r, \Omega(z, w)) \leq(\lambda+o(1)) T(r, w)
$$

on a set of logarithmic density 1 , where $\lambda=\max _{i \in(i)}\left\{\sum_{l=0}^{n} i_{l}\right\}$.

Proof. In calculating the proximity function of $\Omega(z, w)$, we split the region of integration into two parts.

By defining

$$
E_{1}=\left\{\varphi \in[0,2 \pi]:\left|w\left(r e^{i \varphi}\right)\right|<1\right\}
$$

and

$$
E_{2}=[0,2 \pi] \backslash E_{1}
$$

We have

$$
2 \pi m(r, \Omega(z, w))=\int_{E_{1}} \log ^{+}|\Omega| d \varphi+\int_{E_{2}} \log ^{+}|\Omega| d \varphi
$$


First, we consider $E_{1}$. Each term of $\Omega(z, w)$ is of the form

$$
a_{(i)}(z) w(z)^{i_{0}}\left(w\left(q_{1} z\right)\right)^{i_{1}} \cdots\left(w\left(q_{n} z\right)\right)^{i_{n}}
$$

and so, writing with $(i)=\left(i_{0}, \ldots, i_{n}\right)$

$$
\Omega(z, w)=\sum_{(i) \in I} a_{(i)}(z) w(z)^{i_{0}}\left(w\left(q_{1} z\right)\right)^{i_{1}} \cdots\left(w\left(q_{n} z\right)\right)^{i_{n}} .
$$

For each $i$, we have

$$
\begin{aligned}
\left|\Omega_{(i)}(z, w)\right| & =\left|a_{(i)}(z) w(z)^{i_{0}}\left(w\left(q_{1} z\right)\right)^{i_{1}} \cdots\left(w\left(q_{n} z\right)\right)^{i_{n}}\right| \\
& \leq\left|a_{(i)}\left(r e^{i \varphi}\right)\right|\left|\frac{w\left(q_{1} r e^{i \varphi}\right)}{w\left(r e^{i \varphi}\right)}\right|^{i_{1}} \cdots\left|\frac{w\left(q_{n} r e^{i \varphi}\right)}{w\left(r e^{i \varphi}\right)}\right|^{i_{n}} .
\end{aligned}
$$

Thus, for each $i$, we obtain

$$
\frac{1}{2 \pi} \int_{E_{1}} \log ^{+}\left|\Omega_{(i)}\right| d \varphi \leq m\left(r, a_{(i)}\right)+O\left(\sum_{j=1}^{n} m\left(r, \frac{w\left(q_{j} z\right)}{w(z)}\right)\right) .
$$

Since

$$
T\left(r, w\left(q_{j} z\right)\right)=(1+o(1)) T(r, w),
$$

by Lemma 2.1 and (2.2), we obtain

$$
\frac{1}{2 \pi} \int_{E_{1}} \log ^{+}|\Omega| d \varphi=o(T(r, w))
$$

on a set of logarithmic density 1.

On $E_{2}$, we have

$$
\begin{aligned}
|\Omega(z, w)| & =\sum_{(i) \in I} a_{(i)}(z) w(z)^{i_{0}}\left(w\left(q_{1} z\right)\right)^{i_{1}} \cdots\left(w\left(q_{n} z\right)\right)^{i_{n}} \mid \\
& \leq \sum_{(i) \in I}\left|a_{(i)}\left(r e^{i \varphi}\right)\right|\left|w\left(r e^{i \varphi}\right)\right|^{\lambda_{1}}\left|\frac{w\left(q_{1} r e^{i \varphi}\right)}{w\left(r e^{i \varphi}\right)}\right|^{i_{1}} \cdots\left|\frac{w\left(q_{n} r e^{i \varphi}\right)}{w\left(r e^{i \varphi}\right)}\right|^{i_{n}},
\end{aligned}
$$


where $\lambda=\max \left\{i_{0}+\cdots+i_{n}\right\}$, and so, by Lemma 2.1 again,

$$
\frac{1}{2 \pi} \int_{E_{2}} \log ^{+}|\Omega| d \varphi \leq \lambda m(r, w)+o(T(r, w))
$$

on a set of logarithmic density 1.

Combining (2.3) and (2.5), we have

$$
\frac{1}{2 \pi} \int_{E} \log ^{+}|\Omega| d \varphi \leq \lambda m(r, w)+o(T(r, w))
$$

on a set of logarithmic density 1.

Next, we prove

$$
N(r, \Omega(r, w)) \leq \lambda N(r, w)+o(T(r, w))
$$

on a set of logarithmic density 1 .

Let $\Omega_{(i)}=a_{(i)}(z)(w(z))^{i_{0}}\left(w\left(q_{1} z\right)\right)^{i_{1}} \cdots\left(w\left(q_{n} z\right)\right)^{i_{n}}$. It follows from Lemma 2.2 that

$$
\begin{aligned}
N\left(r, \Omega_{(i)}\right) \leq & N\left(r, a_{(i)}(z)\right)+i_{0} N(r, w(z))+i_{1} N\left(r,\left(q_{1} z\right)\right)+\cdots+i_{n} N\left(r, w\left(q_{n} z\right)\right) \\
\leq & N\left(r, a_{(i)}(z)\right)+i_{0} N(r, w(z))+i_{1} N(r, w(z))+\cdots+i_{n} N(r, w(z)) \\
& +o(T(r, w)) \\
= & N\left(r, a_{(i)}(z)\right)+\left[i_{0}+i_{1}+\cdots+i_{n}\right] N(r, w(z))+o(T(r, w)) .
\end{aligned}
$$

Further, we obtain

$$
N(r, \Omega(z, w)) \leq \lambda N(r, w)+o(T(r, w))
$$

on a set of logarithmic density 1 , where $\lambda=\max _{i \in(i)}\left\{\sum_{l=0}^{n} i_{l}\right\}$.

(2.1) follows by combining (2.6) and (2.8). This completes the proof of Lemma 2.3. 
Lemma $2.4([15])$. Let $P(z, w)=\sum_{i=0}^{p} a_{i}(z) w^{i}$ be polynomial in $w(z)$ with the meromorphic coefficients $\left\{a_{i}(z)\right\}$. If $w(z)$ is a meromorphic function, then

$$
\begin{gathered}
T(r, P(z, w))=p T(r, w)+O\left\{\sum_{i=0}^{p} T\left(r, a_{i}\right)\right\}, \\
T(r, P(z, w)) \geq p\left(T(r, w)-\sum_{i=0}^{p} T\left(r, a_{i}\right)\right)+O(1) .
\end{gathered}
$$

Lemma 2.5 ([12]). Let $\psi:\left[r_{0},+\infty\right) \rightarrow(0,+\infty)$ be positive and bounded in every finite interval. Suppose that

$$
\psi\left(\mu r^{m}\right) \leq A \psi(r)+B,\left(r \geq r_{0}\right),
$$

where $\mu>0, m>1, A>1$ and $B$ are real constants. Then

$$
\psi(r)=O\left((\log r)^{\alpha}\right)
$$

where

$$
\alpha=\frac{\log A}{\log m} .
$$

Lemma 2.6 ([16]). Let $w$ be a transcendental meromorphic function and $p(z)=a_{k} z^{k}+a_{k-1} z^{k-1}+\cdots+a_{1} z+a_{0}, a_{k} \neq 0, k \geq 1$, be a polynomial of degree $k$. Given $0<\delta<\left|a_{k}\right|$, let $\lambda=\left|a_{k}\right|+\delta, \mu=\left|a_{k}\right|-\delta$. Then, given $\varepsilon>0$, for any $a \in \mathbf{C} \cup\{\infty\}$ and for $r$ large enough, we have

$$
\begin{gathered}
k n\left(\mu r^{k}, \frac{1}{w-a}\right) \leq n\left(r, \frac{1}{w(p)-a}\right) \leq k n\left(\lambda r^{k}, \frac{1}{w-a}\right), \\
N\left(\mu r^{k}, \frac{1}{w-a}\right)+O(\log r) \leq N\left(r, \frac{1}{w(p)-a}\right) \leq N\left(\lambda r^{k}, \frac{1}{w-a}\right)+O(\log r), \\
(1-\varepsilon) T\left(\mu r^{k}, w\right) \leq T(r, w(p)) \leq(1+\varepsilon) T\left(\lambda r^{k}, w\right) .
\end{gathered}
$$




\section{Proof of Theorem 1.1}

It follows from (1.1), Lemma 2.3 and Lemma 2.4 that

$$
m T(r, w(p(z)))-m \sum_{j=0}^{m} T\left(r, a_{j}\right) \leq(\lambda+\varepsilon) T(r, w),
$$

i.e.,

$$
m T(r, w(p(z))) \leq(\lambda+\varepsilon) T(r, w)+m \sum_{j=0}^{m} T\left(r, a_{j}\right) .
$$

Combining (3.1) and

$$
T\left(r, a_{i}\right)<K T\left(r^{s}, w\right), \quad i=0,1,2, \cdots, m,
$$

we obtain

$$
T(r, w(p(z))) \leq \frac{\lambda+\varepsilon}{m} T(r, w)+(m+1) K T\left(r^{s}, w\right),
$$

where $K$ is a positive constant.

Case (1). If $s \geq 1$, by ([12], pp. 35), we have $\frac{T(r, w)}{\log r}$ is increasing functions of $r$, we can obtain for any positive constant $C$ and any $t \geq 1$,

$$
\frac{T\left(C r^{t}, w\right)}{T(r, w)} \geq \frac{\log C+t \log r}{\log r}>(1-\varepsilon) t
$$

Hence, for $r$ sufficiently large,

$$
T(r, w)<\frac{1}{(1-\varepsilon) t} T\left(C r^{t}, w\right) .
$$

Let $s=t, C=1$. Then

$$
T(r, w)<\frac{1}{(1-\varepsilon) s} T\left(r^{s}, w\right)
$$


It follows from (3.2) and (3.4) that

$$
\begin{aligned}
T(r, w(p)) & \leq(m+1) K T\left(r^{s}, w\right)+\frac{\lambda+\varepsilon}{(1-\varepsilon) m s} T\left(r^{s}, w\right) \\
& \leq\left((m+1) K+\frac{\lambda}{m s}+\varepsilon_{1}\right) T\left(r^{s}, w\right) .
\end{aligned}
$$

By Lemma 2.5,

$$
(1-\varepsilon) T\left(\mu r^{k}, w\right) \leq\left((m+1) K+\frac{\lambda}{m s}+\varepsilon_{1}\right) T\left(r^{s}, w\right) .
$$

From the above inequality, we further get

$$
(1-\varepsilon) T\left(\mu r^{\frac{k}{s}}, w\right) \leq\left((m+1) K+\frac{\lambda}{m s}+\varepsilon_{2}\right) T(r, w) .
$$

Since $k>s$, then by (3.14) and Lemma 2.5, we obtain

$$
T(r, w(z))=O\left((\log r)^{\alpha_{1}+\varepsilon}\right)
$$

where

$$
\alpha_{1}=\frac{\log \left((m+1) K+\frac{\lambda}{m s}\right)}{\log \frac{k}{s}} .
$$

Case (2). If $s<1$, by ([12], pp. 35), since $\frac{T(r, w)}{\log r}$ is increasing function of $r$, we obtain

$$
\frac{T(r, w)}{\log r} \geq \frac{T\left(r^{s}, w\right)}{\log r^{s}},
$$

i.e.,

$$
\frac{T(r, w)}{T\left(r^{s}, w\right)} \geq \frac{1}{s}
$$


From (3.2) and (3.7), we get

$$
T(r, w(p(z))) \leq\left(\frac{\lambda+m(m+1) K s+\varepsilon_{3}}{m}\right) T(r, w) .
$$

According to Lemma 2.6, we obtain

$$
T\left(\mu r^{k}, w\right) \leq\left(\frac{\lambda+m(m+1) K s+\varepsilon_{4}}{m}\right) T(r, w) .
$$

We obtain from Lemma 2.5

$$
T(r, w(z))=O\left((\log r)^{\alpha_{2}+\varepsilon}\right)
$$

where

$$
\alpha_{2}=\frac{\log \frac{\lambda+m(m+1) K s}{m}}{\log k} .
$$

Combining case (1) and case (2), we get the proof of Theorem 1.1.

\section{Competing Interests}

The authors declare that they have no competing interests.

\section{Author's Contributions}

All authors drafted the manuscript, read and approved the final manuscript.

\section{Acknowledgements}

The work is supported by the National Natural Science Foundation of China (10471065), and also supported by Key Project of Science and Research of Hebei University of Economics and Business (2017KYZ04). 


\section{References}

[1] H. X. Yi and C. C. Yang, Theory of the Uniqueness of Meromorphic Functions (in Chinese), Beijing: Science Press, 1995.

[2] I. Laine, Nevanlinna Theory and Complex Differential Equations, Walter de Gruyter, Berlin, 1993.

[3] Z. X. Chen and K. H. Shon, On zeros and fixed points of differences of meromorphic functions, J. Math. Anal. Appl. 344(1) (2008), 373-383.

DOI: https://doi.org/10.1016/j.jmaa.2008.02.048

[4] Y. M. Chiang and S. J. Feng, On the Nevanlinna characteristic of $f(z+\eta)$ and difference equations in the complex plane, Ramanujan J. 16(1) (2008), 105-129.

DOI: https://doi.org/10.1007/s11139-007-9101-1

[5] I. Laine, J. Rieppo and H. Silvennoinen, Remarks on complex difference equations, Comput. Methods Funct. Theory 5(1) (2005), 77-88.

DOI: https://doi.org/10.1007/BF03321087

[6] R. G. Halburd and R. J. Korhonen, Difference analogue of the lemma on the logarithmic derivative with applications to difference equations, J. Math. Anal. Appl. 314(2) (2006), 477-487.

DOI: https://doi.org/10.1016/j.jmaa.2005.04.010

[7] R. Korhonen, A new Clunie type theorem for difference polynomials, J. Difference Equ. Appl. 17(3) (2011), 387-400.

DOI: https://doi.org/10.1080/10236190902962244

[8] Y. Wang, Meromorphic solutions of some types of complex differential-difference equations, Acta Mathematica Scientia 37(3) (2017), 732-751.

DOI: https://doi.org/10.1016/S0252-9602(17)30034-6

[9] L. Y. Gao, On meromorphic solutions of a type of difference equations, Chinese Ann. Math. Ser. A 35(2) (2014), 193-202.

[10] L. Y. Gao, The growth order of solutions of systems of complex difference equations, Acta Mathematica Scientia 33(3) (2013), 814-820.

DOI: https://doi.org/10.1016/S0252-9602(13)60040-5

[11] L. Y. Gao, On entire solutions of two types of systems of complex differentialdifference equations, Acta Mathematica Scientia 37(1) (2017), 187-194.

DOI: https://doi.org/10.1016/S0252-9602(16)30124-2

[12] H. Silvennoinen, Meromorphic solutions of some composite functional equations, Ann. Acad. Sci. Fenn. Math. Diss. 133 (2003), 1-39. 
[13] D. C. Barnett, R. G. Halburd, R. J. Korhonen and W. Morgan, Nevanlinna theory for the $q$-difference operator and meromorphic solutions of $q$-difference equations, Proc. Roy. Soc. Edinburgh Sect. A 137(3) (2007), 457-474.

DOI: https://doi.org/10.1017/S0308210506000102

[14] J. L. Zhang and R. Korhonen, On the Nevanlinna characteristic of $f(q z)$ and its applications, J. Math. Anal. Appl. 369(2) (2010), 537-544.

DOI: https://doi.org/10.1016/j.jmaa.2010.03.038

[15] A. Z. Mokhon'ko and V. D. Mokhon'ko, Estimates for the Nevanlinna characteristics of some classes of meromorphic functions and their applications to differential equations, Sib. Math. J. 15(6) (1974), 921-934.

DOI: https://doi.org/10.1007/BF00966560

[16] R. Goldstein, Some results on factorisation of meromorphic functions, J. Lond. Math. Soc. 4(2) (1971), 357-364.

DOI: https://doi.org/10.1112/jlms/s2-4.2.357 